\title{
Spin Zeros and the Origin of Fermi Surface Reconstruction in the Cuprates
}

\author{
M. R. Norman and Jie Lin \\ Materials Science Division, Argonne National Laboratory, Argonne IL 60439
}

(Dated: August 9, 2021)

\begin{abstract}
Two recent quantum oscillation studies find contradictory results concerning the existence of spin zeros - zeros of the oscillatory signal induced by Zeeman splitting of the Landau levels. We discuss these experiments in light of calculations of the oscillations assuming a spin density wave state. We find that the lack of spin zeros reported in one of the experiments is consistent with either hole or electron pockets in such a state, if the staggered moment is perpendicular to the external field. An analysis for field directions near the planes might be able to differentiate between the two. On the other hand, if spin zeros exist as reported in the other experiment, then the staggered moment would have to have a substantial longitudinal component. We suggest several experiments to test whether this is indeed the case.
\end{abstract}

PACS numbers: $74.25 . J b, 72.15 . \mathrm{Gd}, 75.30 . \mathrm{Fv}$

Since the pioneering work of Doiron-Leyraud et al.,1 quantum oscillation studies have provided new insights into the nature of the electronic ground state of the cuprates. For overdoped compounds, a large Fermi surface is observed, $\stackrel{2]}{2}$ consistent with previous photoemission studies ${ }^{33}$ and paramagnetic band structure calculations. $\frac{4}{4}$ For underdoped compounds, this Fermi surface breaks up into smaller pockets. ${ }^{[15 ! 7}$ It is thought that this break up is due to a density wave reconstruction of the Fermi surface, perhaps due to the formation of magnetic stripes $\left[\frac{819}{}\right.$ These findings have had a significant impact on our understanding of the cuprate phase diagram, $\frac{10}{1}$ though debates continue about the relation of these small pockets to the Fermi 'arcs' observed in zero field photoemission studies.

A key insight into the nature of this underdoped high field ground state was recently provided by Sebastian et al. ${ }^{11}$ They looked at the oscillations as a function of the angle of the magnetic field relative to the crystallographic $c$-axis. If Zeeman splitting were present, then such a rotation study should find certain angles, known as spin zeros, where the oscillation amplitude would go through zero, with higher angles exhibiting a $\pi$ phase shift relative to lower ones. Up to an angle near $60^{\circ}$, they did not observe this effect. This was confirmed in a more extensive study ${ }^{7}$ As they suggest, such a finding would be consistent with the presence of a spin density wave state ${ }^{\frac{819112}{12}}$ as we will elaborate on below. Subsequent to this, though, another quantum oscillation study was done where zeros from two different frequencies were found from fits to the data, with one near $40^{\circ}$ and the other near $50^{\circ} ! 13$ Based on this, the authors suggest a paramagnetic ground state instead.

These results raise a general question about the nature of the high field ground state, and how much the Zeeman splitting is actually reduced if this ground state is a spin density wave state. Recently, Ramazashvili, 14 following an earlier study by Kabanov and Alexandrov, 15 has shown on symmetry ground $\$ \frac{16}{6}$ that for commensurate spin density wave ordering, $Q=(\pi, \pi)$, the Zeeman splitting should be quenched for both electron and hole pockets, as they are centered at the magnetic zone boundary. This is under the assumption that the magnetic field strength is large enough for the spins to be reoriented perpendicular to the field. On the other hand, for the incommensurate (stripe) case, only the hole pockets have this symmetry. His suggestion was this finding could be used to resolve the controversy of whether the observed oscillation signal originated from electron or hole pockets.

In this Rapid Communication, we perform calculations for a magnetic stripe in the presence of an external field. We verify that in the configuration where the spin moments are transverse to the field, the hole pockets do not exhibit any Zeeman splitting. On the other hand, we find that the Zeeman splitting of the electron pockets is strongly reduced compared to that of a paramagnetic ground state. Implications of our results in regards to the quantum oscillation experiments, and proposals for new experiments, will be discussed.

Our model assumes a linear spin density wave state $[9$ Without an external field, the secular matrix has dimension $N$, where the magnetic ordering wavevector is $Q=(1-2 / N, 1) \pi$. Diagonal elements of this matrix are of the form $\epsilon_{k+n Q}$ with $n$ running from 1 to $N$, where $\epsilon_{k}$ is the paramagnetic dispersion. Off-diagonal elements due to the stripe order are of the form $V_{s} \delta_{n, n \pm 1}$ and $V_{c} \delta_{n, n \pm 2}$ where $V_{s}$ is the magnetic potential, and $V_{c}$ its charge harmonic (for simplicity, we ignore higher harmonics).

In the presence of an external field, spin must be explicitly taken into account, leading to a secular matrix of dimension $2 N$. Taking the spin quantization axis to be along the field direction, the Zeeman splitting entering into the diagonal terms would be $\frac{g}{2} \mu_{B} H \sigma_{z}$ where $g$ is the $\mathrm{g}$ factor, $\mu_{B}$ the Bohr magneton, $H$ the external field, and $\sigma_{z}$ a Pauli matrix. The term $V_{c}$ would have a $\sigma_{0}$ matrix associated with it instead. Ignoring small effects due to spin-orbit coupling except for their renormalization of $g$, then only the relative orientation of the staggered moment and the field is important. Thus, $V_{s}$ becomes $V_{s}\left(\sigma_{x} \sin \phi+\sigma_{z} \cos \phi\right)$ where $\phi$ is the angle between the staggered moment and the field. For $\phi=0$, the staggered moment is longitudinal, and for $\phi=\pi / 2$ 
it is transverse. We note that for both the longitudinal and transverse cases, the secular matrix decomposes into two diagonal blocks with dimension $N$. The 'down' spin block can be obtained from the 'up' spin block by inverting the sign of $H$ (and $V_{s}$ in the longitudinal configuration). For the transverse case, the 'down' spin eigenvalue spectrum is equivalent to the 'up' spin one if $k$ is translated by $Q$. For the longitudinal case, with $V_{c}=0$, the secular matrices are equivalent to the paramagnetic case except for a shift of $\epsilon_{k}$ by $\pm \frac{g}{2} \mu_{B} H$ (and thus one has full Zeeman splitting). The transverse case is more interesting; detailed calculations presented below find that the Zeeman splitting is strongly suppressed. For each $H$, we determine the chemical potential to obtain the correct occupation number (which we take to be $1 / N$ holes).

In Fig. 1a, we show the Fermi surface for $N=8$ in the transverse configuration, with an artificially large value of $H$ so as to emphasize the Zeeman splitting. The effect of the Zeeman field is to split each of the two hole pockets (which have equal area) by shifting their centers relative to one another. But the split pockets have the same area, as they are related to one another by the symmetries of the underlying Hamiltonian. This is associated with the fact that the Zeeman splitting is identically zero at the paramagnetic orbit centers, as previously derived by Ramazashvili, 14 which he denotes as points $S \equiv([2 n+1] / N, 1 / 2) \pi$. To see this in greater detail, we plot in Fig. $1 \mathrm{~b}$ the zero contours of $E_{n \uparrow}-E_{n \downarrow}$, where $E_{n \sigma}$ are the eigenvalues of the secular matrix. For this plot, an experimentally relevant value of $H$ is chosen instead. One can indeed see that the zero contours for each band all intersect at the points $S$. On the other hand, these zero contours are not all confined to being near the zone boundary $k_{y} \sim \pi / 2$. In particular, zero contours cut across the electron pockets, and as a consequence, even though the two split electron pockets have different areas, this difference is much smaller than would occur in the longitudinal case. We have not been able to identify any symmetry reason for why this occurs. Plots of the dispersion along the symmetry axis $k_{y}=0$ reveal that for the electron band (but not for most of the other bands), the Zeeman splitting is very small at the bottom of the band, the so-called $B$ points $\frac{14}{14}$ at $(2 n / N, n-1) \pi$.

We contrast this with the $N=10$ case shown in Fig. 2 . The fundamental difference here is that in the paramagnetic case, there are now two hole bands, each with the same pocket area. Again, the hole pockets are split by the Zeeman term, maintaining equal areas. But interestingly, all bands have a zero contour for $E_{n \uparrow}-E_{n \downarrow}$ equal to the $k_{y}=\pi / 2$ line. Related to this is the fact that the paramagnetic orbit centers of the two hole pockets (and related cases with $N$ differing by 4 ) are not staggered like for the $N=8$ case, but are degenerate (again, this degeneracy is connected to the presence of two hole bands as compared to the single hole band of the $N=8$ case). These points are of the form $(4 n / N, 1 / 2) \pi$. Interestingly, the degeneracy present along this horizontal line $\left(k_{y}=\pi / 2\right)$ does not occur along any vertical lines.
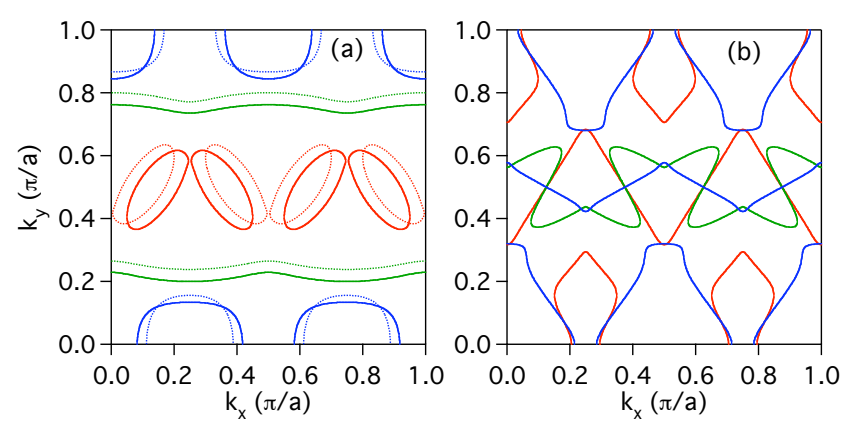

FIG. 1: (Color online) $N=8$ transverse case with $V_{s}=0.178$ $\mathrm{eV}, V_{c}=0$. (a) Fermi surfaces for $\frac{g}{2} H=1000$ Tesla (this is artificially large so as to emphasize the Zeeman splitting). Solid and dotted curves represent the Fermi surfaces from the 'up' and 'down' spin bands. (b) zero contours of $E_{n \uparrow}-E_{n \downarrow}$, where $E_{n \sigma}$ are the eigenvalues of the secular matrix with $n$ the band index and $\sigma$ the spin. Here, $\frac{g}{2} H$ is taken to be 60 Tesla.
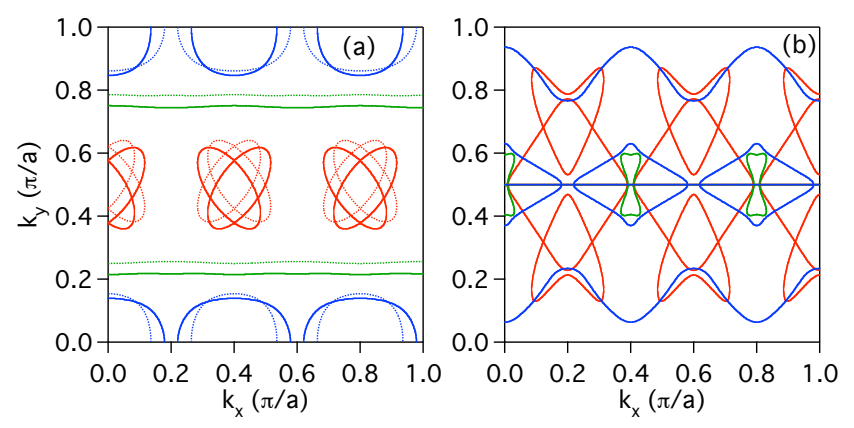

FIG. 2: (Color online) $N=10$ transverse case with $V_{s}=0.204$ $\mathrm{eV}, V_{c}=0$. (a) Fermi surfaces for $\frac{g}{2} H=1000$ Tesla (this is artificially large so as to emphasize the Zeeman splitting). Solid and dotted curves represent the Fermi surfaces from the 'up' and 'down' spin bands. (b) zero contours of $E_{n \uparrow}-E_{n \downarrow}$, where $E_{n \sigma}$ are the eigenvalues of the secular matrix with $n$ the band index and $\sigma$ the spin. Here, $\frac{g}{2} H$ is taken to be 60 Tesla.

We now calculate the Zeeman splitting of the electron pocket for the $N=8$ and 10 cases, both for the longitudinal and transverse configurations. $V_{s}$ was chosen to yield an orbit frequency (without Zeeman splitting) for the electron pocket near 530 Tesla as observed experimentally ${ }^{17}$ As we use a tight binding fit to band theory for $\epsilon_{k},{ }^{4}$ we also ran these calculations with a mass renormalization, $Z$, of 3 to come into better agreement with the observed cyclotron mass. This renormalization is imposed not only on $\epsilon_{k}$, but on $V_{s}$ and $V_{c}$ as well to maintain the pocket size. Obviously, the Zeeman effect is amplified as $Z$ is increased. Our results are summarized in Table I. There, it can be seen that the Zeeman splitting is drastically reduced in the transverse case, by factors which range from 7.5 to 20 relative to the longitudinal case where full Zeeman splitting is observed. We chose to do the calculations for a field of 60 Tesla to maximize 
TABLE I: Splitting of the electron orbit frequencies for an applied field, $\frac{g}{2} H$, of 60 Tesla. $N$ is the stripe period, $Z$ the mass renormalization, and $P$ the polarization of the spins $(L$ for the spins aligned with the field, $T$ for the spins perpendicular to the field). The quantity $2 \Delta F / H$, where $\Delta F$ is the frequency splitting, is equivalent to $g m_{s} / m$ where $g$ is the gfactor and $m_{s}$ the 'spin' mass. For $N=8, V_{s}$ is $0.178 \mathrm{eV}$; for $N=10, V_{s}$ is $0.204 \mathrm{eV} 17$

\begin{tabular}{cccc}
\hline \hline $\mathrm{N}$ & $\mathrm{Z}$ & $\mathrm{P}$ & $2 \Delta F / H$ \\
\hline 8 & 1 & $\mathrm{~L}$ & 1.19 \\
& & $\mathrm{~T}$ & 0.06 \\
& 3 & $\mathrm{~L}$ & 3.55 \\
& & $\mathrm{~T}$ & 0.19 \\
10 & & $\mathrm{~L}$ & 1.30 \\
& & $\mathrm{~T}$ & 0.17 \\
& & $\mathrm{~L}$ & 3.90 \\
& \multirow{3}{*}{3} & $\mathrm{~T}$ & 0.52 \\
\hline \hline
\end{tabular}

the Zeeman effect for physically relevant fields. For the $N=8$ case in the transverse configuration, we have verified that the frequency splitting of the orbits is linear in $H$ up to 60 Tesla.

To compare to experiment, we need to estimate the effect the splitting has on the oscillation amplitude. Following Lifshitz and Kosevich, 18 one finds that the Zeeman reduction in the amplitude, $R_{s}$, can be written as $\cos (p \pi \Delta F /[H \cos \theta])$ where $p$ is the harmonic index, $\Delta F$ the difference of the Zeeman split orbit areas (expressed as a cyclotron frequency), and $\theta$ the angle of the field relative to the $c$-axis. Comparing to the standard formula for $R_{s}$, we then equate $2 \Delta F / H$ with $g m_{s} / m$ where $m_{s}$ is the 'spin' mass. This is the quantity typically reported by experiment.

In Figs. 3 and 4 , we show $R_{s}$ for the electron pocket for the $N=8$ and 10 cases, for $Z=1$ and 3 , and also for both longitudinal and transverse configurations (for the fundamental harmonic $p=1$ ). For the transverse case, spin zeros typically occur at very high angles due to the drastically reduced Zeeman splitting in this case. This can be contrasted with the longitudinal case, where full Zeeman splitting occurs resulting in values comparable to a paramagnetic state. We remind that for the hole pockets, $R_{s}$ would be identically 1 for the transverse case.

We now turn to comparing our results to experiment. Sebastian et al! 11 find no spin zeros up to angles near $60^{\circ}$, nor any evidence for a $\pi$ phase shift of the oscillations that would be consistent with passing through such a zero. This would definitely be consistent with a hole pocket as commented on previously by Ramazashvili, 14 but could be consistent with electron pockets as well. To know for sure would require extending the measurements to higher angles, or analyzing higher harmonics. ${ }^{[19}$ Unfortunately, this is a quantitative issue, since we have shown one case in Table I where $2 \Delta F / H$ is as small as 0.06 , and this could be potentially smaller for different choices of
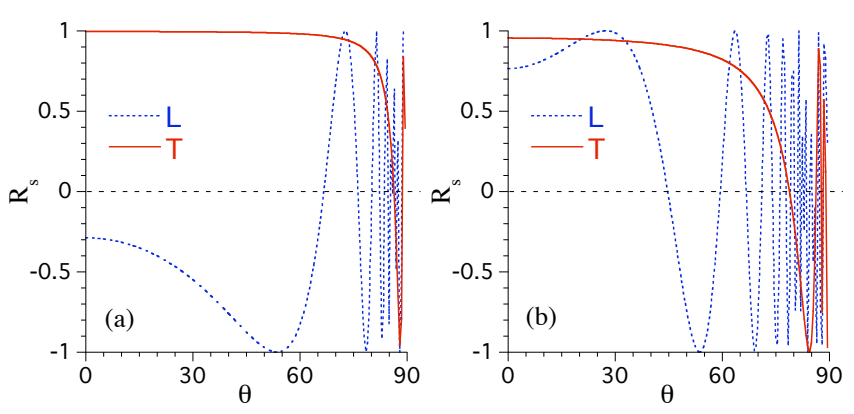

FIG. 3: (Color online) Spin amplitude factor $R_{s}$ versus angle of the field relative to the c-axis with $\frac{g}{2} H=60$ Tesla and $N=8$ for longitudinal $(L)$ and transverse $(T)$ spins. (a) $Z=1$ and (b) $Z=3$, where $Z$ is the mass renormalization. Same parameters as Fig. 1b.
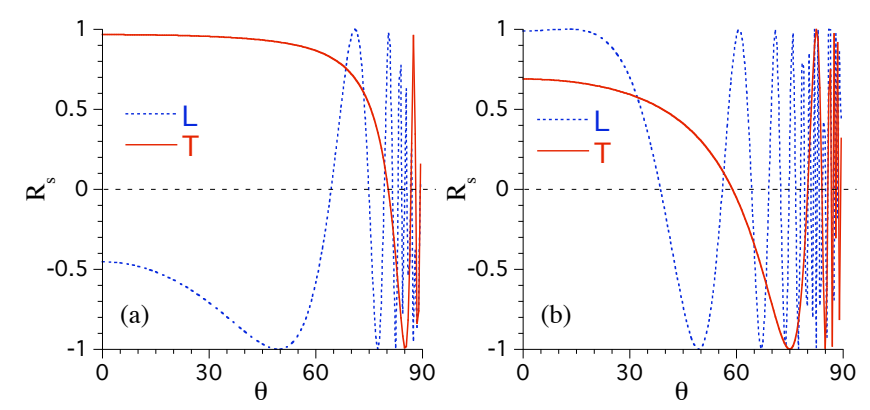

FIG. 4: (Color online) Spin amplitude factor $R_{s}$ versus angle of the field relative to the c-axis with $\frac{g}{2} H=60$ Tesla and $N=10$ for longitudinal $(L)$ and transverse $(T)$ spins. (a) $Z=1$ and (b) $Z=3$, where $Z$ is the mass renormalization. Same parameters as Fig. $2 b$.

$V_{s}$ and $V_{c}$.

In contrast, Ramshaw et al ${ }^{13}$ were motivated to include an $R_{s}$ factor in their fits in an attempt to describe the non-trivial dependence of the overall oscillation amplitude on the field angle. From their fits, they claim zeros at $50^{\circ}\left(\mathrm{gm}_{\mathrm{s}} / \mathrm{m}=3.2\right)$ for the fundamental frequency and $40^{\circ}\left(\mathrm{gm}_{\mathrm{s}} / \mathrm{m}=2.1\right)$ for a smaller secondary frequency. For the fundamental frequency, $R_{s}$ initially increases as $\theta$ increases from 0 . This would only be consistent with a paramagnetic state, or a density wave state of nonmagnetic origin, such as the $d$-density wave state. ${ }^{20} \mathrm{It}$ is also consistent with the spin density wave scenario if we assume that the staggered moment has a large component along the field direction.

Noting that these two measurements are different (Ramshaw et al. measure the c-axis resistance, whereas Sebastian et al. measure the change in the skin depth), the source of the discrepancy between the two has to do with the complex waveform of the oscillations. Even if there were only one pocket present, up to four frequencies could be realized due to (1) bilayer splitting and (2) warping of each individual cylinder (leading to extremal neck and belly frequencies). In the analysis of Ref. 11, the authors did not detect any amplitude suppression or 
phase shift that would be consistent with the presence of a spin zero. They verified this in more extensive work where they also did not detect any such effect in the second harmonic where it would have been more obvious. ${ }^{7}$ On the other hand, Ramshaw et al ${ }^{[13}$ claim that the presence of closely spaced frequencies can mask the presence of zeros for each individual frequency. Their analysis involved a fit including both the primary and a lower secondary frequency, though they did not include the higher secondary frequency claimed in other measurements 6$]$

To make further progress would require detailed fits including all three frequencies over a wider field range. Moreover, these measurements should be supplemented by NMR, neutron, and magneto-resistance data to look for the orientation of the spins in the high field state. So far, little is known about this for underdoped $\mathrm{YBa}_{2} \mathrm{Cu}_{3} \mathrm{O}_{y}$ samples in the doping range magnetic oscillations have been observed. For a lower doping, a field induced elastic signal has been observed by neutrons, 21 but the orientation of the spins relative to the field has not been determined. Several studies, though, have been made on $1 / 8$ doped $\mathrm{La}_{2-x} \mathrm{Ba}_{x} \mathrm{CuO}_{4}$, where a magnetic stripe state is stable at zero field. What is observed from bulk susceptibility studies is a spin flop field of about 6
Tesla, above which the spins appear to be rotated transverse to the applied field. ${ }^{22}$ Based on this, we feel it quite likely for the extremely high fields used in the magnetic oscillation studies, the spins are indeed in the transverse configuration. But further studies will be necessary to see whether this is indeed the case for $\mathrm{YBa}_{2} \mathrm{Cu}_{3} \mathrm{O}_{y}$ with $y \sim 6.5$. In the quantum oscillation context, this could be tested by comparing angle sweeps in the $a c$ and $b c$ planes, assuming the spins are locked to a particular axis in the plane as occurs for $1 / 8$ doped $\mathrm{La}_{2-x} \mathrm{Ba}_{x} \mathrm{CuO}_{4}$ at low fields. We note that Sebastian et al. did not find any significant changes of their data as a function of the in-plane field angle.

We thank Revaz Ramazashvili, Andy Millis, Cyril Proust, Brad Ramshaw, Neil Harrison and Suchitra Sebastian for extensive discussions concerning the spin zeros. We are particularly indebted to Suchitra Sebastian for sharing with us some of her unpublished analysis with Gil Lonzarich. This work was supported by the US DOE, Office of Science, under contract DE-AC02-06CH11357 and by the Center for Emergent Superconductivity, an Energy Frontier Research Center funded by the US DOE, Office of Science, under Award No. DE-AC0298CH1088.
1 N. Doiron-Leyraud, C. Proust, D. LeBoeuf, J. Levallois, J.-B. Bonnemaison, R. Liang, D. A. Bonn, W. N. Hardy and L. Taillefer, Nature 447, 565 (2007).

2 B. Vignolle, A. Carrington, R. A. Cooper, M. M. J. French, A. P. Mackenzie, C. Jaudet, D. Vignolles, C. Proust and N. E. Hussey, Nature 455, 952 (2008).

3 A. Damascelli, Z. Hussain and Z.-X. Shen, Rev. Mod. Phys. 75, 473 (2003).

4 O. K. Andersen, A. I. Liechtenstein, O. Jepsen and F. Paulsen, J. Phys. Chem. Solids 56, 1573 (1995).

5 S. E. Sebastian, N. Harrison, E. Palm, T. P. Murphy, C. H. Mielke, R. Liang, D. A. Bonn, W. N. Hardy and G. G. Lonzarich, Nature 454, 200 (2008).

6 A. Audouard, C. Jaudet, D. Vignolles, R. Liang, D. A. Bonn, W. N. Hardy, L. Taillefer and C. Proust, Phys. Rev. Lett. 103, 157003 (2009).

7 S. E. Sebastian, N. Harrison, P. A. Goddard, M. M. Altarawneh, C. H. Mielke, R. Liang, D. A. Bonn, W. N. Hardy, O. K. Andersen and G. G. Lonzarich, Phys. Rev. B 81, 214524 (2010).

8 D. LeBoeuf, N. Doiron-Leyraud, R. Daou, J.-B. Bonnemaison, J. Levallois, N.E. Hussey, C. Proust, L. Balicas, B. Ramshaw, R. Liang, D.A. Bonn, W.N. Hardy, S. Adachi and L. Taillefer, Nature 450, 533 (2007).

9 A. J. Millis and M. R. Norman, Phys. Rev. B 76, 220503(R) (2007).
10 S. Sachdev, Phys. Status Solidi B 247, 537 (2010).

11 S. E. Sebastian, N. Harrison, C. H. Mielke, R. Liang, D. A. Bonn, W. N. Hardy and G. G. Lonzarich, Phys. Rev. Lett. 103, 256405 (2009).

12 N. Harrison, Phys. Rev. Lett. 102, 206405 (2009).

13 B. J. Ramshaw, B. Vignolle, J. Day, R. Liang, W. N. Hardy, C. Proust and D. A. Bonn, arXiv:1004.0260

14 R. Ramazashvili, arXiv:1006.0167

15 V. V. Kabanov and A. S. Alexandrov, Phys. Rev. B 77, 132403 (2008) and 81, 099907(E) (2010).

16 R. Ramazashvili, Phys. Rev. B 79, 184432 (2009).

17 M. R. Norman, J. Lin and A. J. Millis, Phys. Rev. B 81, 180513(R) (2010).

18 D. Shoenberg, Magnetic oscillations in metals (Cambridge University Press, Cambridge, 1984).

19 Suchitra Sebastian, private communication.

20 S. Chakravarty, R. B. Laughlin, D. K. Morr and C. Nayak, Phys. Rev. B 63, 094503 (2001).

21 D. Haug, V. Hinkov, A. Suchaneck, D. S. Inosov, N. B. Christensen, Ch. Niedermayer, P. Bourges, Y. Sidis, J. T. Park, A. Ivanov, C. T. Lin, J. Mesot and B. Keimer, Phys. Rev. Lett. 103, 017001 (2009).

${ }^{22}$ M. Hucker, G. D. Gu and J. M. Tranquada, Phys. Rev. B 78, 214507 (2008). 